\title{
Relation of space-time continuum with energy in the presence of consciousness and super-consciousness
}

\author{
Dhananjay Pal \\ Email address: \\ dhananjay.pal123@gmail.com,paldhananjay46@yahoo.com
}

Pharmacy College, Bengal School of Technology, Sugandha-Delhi Road, Chuchura, Dist.-Hooghly, West Bengal, INDIA, PIN-712 102

\section{To cite this article:}

Dhananjay Pal. Relation of Space-Time Continuum with Energy in the Presence of Consciousness and Super-Consciousness. American Journal of Physics and Applications. Vol. 1, No. 3, 2013, pp. 67-79. doi: 10.11648/j.ajpa.20130103.14

\begin{abstract}
Our consciousness model involving thought-carrying particle (TCP), thought retaining particle (TRP) and thought force $\left(\mathrm{T}_{\mathrm{F}}\right)$ signifies the existence of universal consciousness that exists along with the universe. This universal consciousness is a functional state of the universal mind. This universal mind (UM) is evolved at the Big Bang from void. The UM is constituted by these TCP and TRP in the inherent presence of thought force $\left(T_{F}\right)$. Thought force ( $\left.T_{F}\right)$ is an expression of universal consciousness. The Thought force $\left(\mathrm{T}_{\mathrm{F}}\right)$ being the primordial quantum field functions as the original super-force. $\mathrm{T}_{\mathrm{F}}$ being the original super-force functions as the origin of all the fundamental fields. TCP is the carrier of thought force $\left(\mathrm{T}_{\mathrm{F}}\right)$ that, in turn, appears to be the origin of all the fields. We show here that time of any kind cannot exist without the existence of consciousness. Time without consciousness is not possible. But consciousness without time is possible. Space and time are dynamic quantities and they not only affect but also are affected by everything that happens in the Universe. As per Hawking, "Time is not completely separate from and independent of space but is combined with it to form what is called space-time continuum". Space can not exist without time and vice versa. Space-time without consciousness is not possible. Space-time exists only in the existence of consciousness. Although space-time continuum is thought to be a four-dimensional object, yet Space and Time appear to be subjective in nature. The quantized energy $\left(\varepsilon_{T}\right)$ of Though-carrying particle (TCP) is responsible to cause the universal consciousness as well as the cosmic microwave background radiation temperature. The individual consciousness owes its origin to the universal consciousness created by the same $\varepsilon_{T}$. The same $\varepsilon_{T}$ is the energy responsible for generating Thought force $\left(\mathrm{T}_{\mathrm{F}}\right) . \mathrm{T}_{\mathrm{F}}$ being an expression of the universal consciousness is applicable to any inanimate object as well as to any biological system (having thinking ability). The $\mathrm{T}_{\mathrm{F}}$ exerts its functions both in vitro and in vivo. The manifestation of any type of time is solely dependent on the generation of consciousness $\left[\left(=\mathcal{E}_{T}=m_{T} c^{2}=h c / \lambda_{T}\right)\right]$. The origin of the physical time appears to be the origin of evolution of light and its evolved velocity (c) at that point of the beginning of universe. The velocity (c) of light as well as time is always related with consciousness. Consciousness is conjectured here to be the mental light.
\end{abstract}

Keywords: Universal Mind (UM), Thought Force $\left(\mathrm{T}_{\mathrm{F}}\right)$, Though-Carrying Particle (TCP), Thought Retaining Particle (TRP), Quantized Energy $\left(\varepsilon_{T}\right)$ of TCP, Cosmic Microwave Background Radiation (CMBR)

\section{Introduction}

In Eastern philosophical traditions, consciousness is intrinsic to the universe, whereas in most Western views, consciousness is extrinsic, emerging from complex computation. How can these views be reconciled?

It is most relevant and important to indicate the names of various eminent physicists like Erwin Schrödinger, Eugene Wigner, Brian Josephson, John Wheeler, Roger Penrose, Henry P Stapp, Freeman J. Dyson, Paul Davies, David Bohm, Basil Hiley, Fritjof Capra, Fred Alan Wolf and Amit
Goswami who have addressed the inclusion of consciousness in their work. Consciousness is to be taken into account.

Consciousness model of Pal et al [1-3] involving TCP, TRP and thought force $\left(\mathrm{T}_{\mathrm{F}}\right)$ signifies the existence of universal consciousness that exists along with the universe.

\subsection{General View for the Evolution of the Universe}

Big Bang $\rightarrow$ Universe $\rightarrow$ Fields + Particles \& Antiparticles + STC $] \rightarrow[$ Matter and Fields + STC $] \rightarrow$ Matter and Fields + STC + Life as well as consciousness.

If the universe exists along with the universal 
consciousness that, in turn, is a functional state of the universal mind (UM) and if this UM is evolved at the Big Bang from the eternal void, then

\subsubsection{Our View for the Evolution of the Universe (See Scheme-I)}

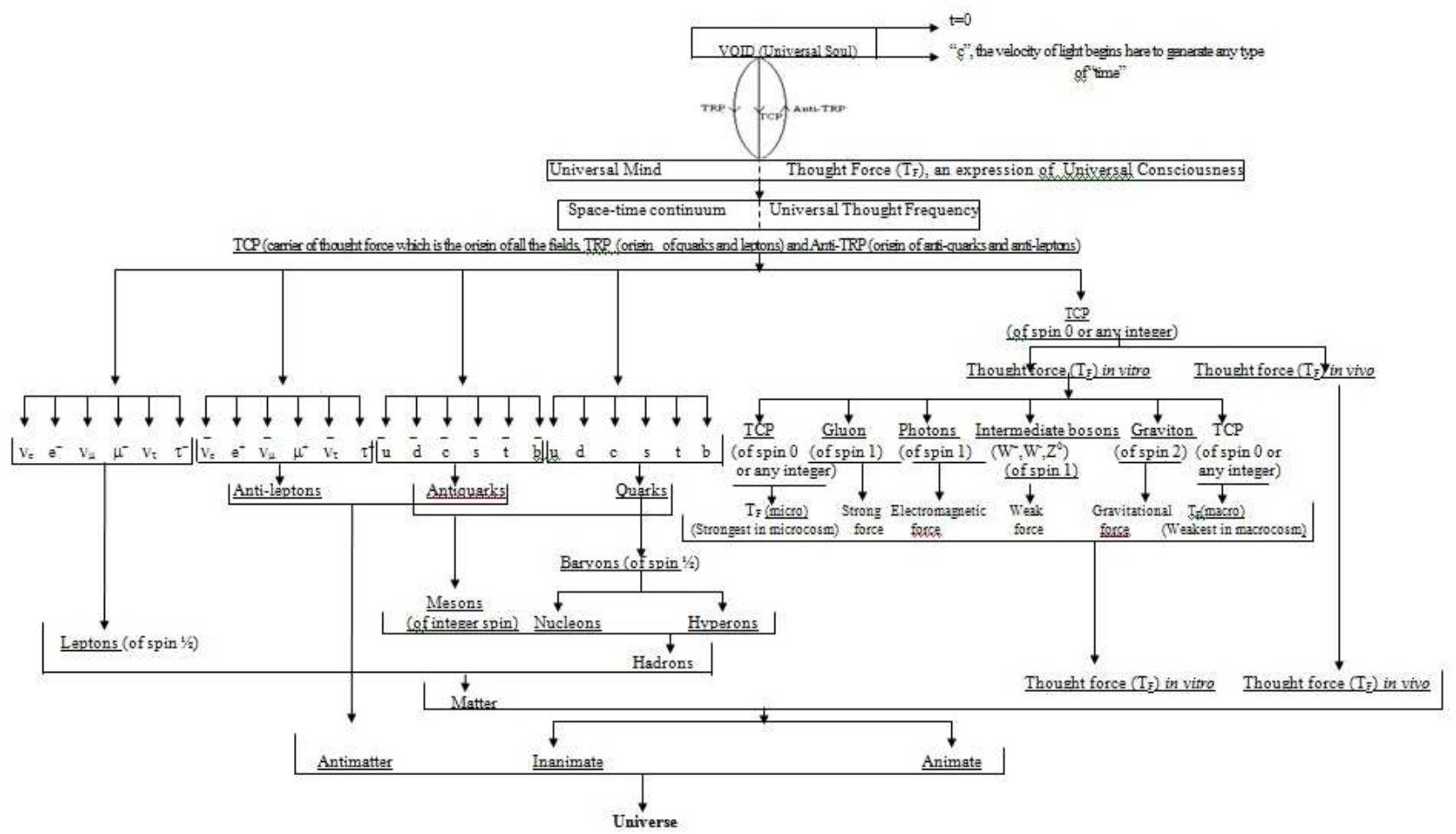

Scheme 1. Schematic representation showing the evolution of the universe from the void incorporating the postulated TCP (Thought carrying particle), TRP(Thought retaining particle), and thought force $\left(T_{F}\right)$. Laws of absolute conservation of mass. Charge (and color) are to be maintained in order to develop a general theory for the unification of physics which would be freely applicable to the more general situations involving the non-living system and living system having consciousness. Here TCP is the carrier of the thought force ( $\left.T_{F}\right)$ that is the origin of all fields. TRP is origin of the all matter particles. It is to be noted that these TCP and TR function like wavicle: wave-particle duality. Here thought force ( $\left.T_{F}\right)$ is an expression of the "universal consciousness"

$\mathrm{VOID} \rightarrow[$ Big Bang $] \rightarrow[\mathrm{TCP}, \mathrm{TRP} \&$ Anti-TRP in the inherent presence of thought force (TF) + STC] $\rightarrow$ [Universal Mind with universal consciousness + TCP \& TRP in the inherent presence of thought force (TF) + STC $+\mathrm{UTF}] \rightarrow$ [Universal Mind with universal consciousness + Matter and Fields \{including thought force (TF) $\}+$ TCP \& $\mathrm{TRP}+\mathrm{STC}+\mathrm{UTF}] \rightarrow$ Universal Mind with universal consciousness + Matter and Fields \{including thought force $(\mathrm{TF})\}+$ Life as well as consciousness + TCP \& TRP + STC + UTF [where thought force (TF) is the origin of all the fields, TCP (Though-carrying particle) is the origin of all the field particles, TRP (Thought retaining particle) is the origin of all the matter particles, anti-TRP is the origin of all the anti-particles, STC $=$ Space time continuum and $\mathrm{UTF}=$ Universal thought frequency $\cong$ frequency of the TCP].

Bhaumik [4] mentioned, "It would be reasonable to presume that the universe originated in a unity of all fields at or near Planck's dimension. John Wheeler strongly believes that "in defining any useful concept of reality" we have to take into account "the indispensable place of the participating observer---evidenced in quantum mechanics," the foregoing cannot be really true unless consciousness is as essential as aspect of nature as are the fields that give rise to force and matter and the primary field that gave rise to them".

Physicists determined that underlying quantum fields give birth to elementary particles. Bhaumik [4] mentioned that Frank Wilczek pointed out, "In quantum field theory, the primary elements of reality are not individual particles, but underlying fields. Thus, for example, all electrons are but excitations of an underlying field, naturally called electric field". The same holds true for all the fundamental particles of which matter is made.

TCP cannot exist without TRP and vice versa. Many physicists believe that unifying all the forces, including gravity, into a single theory would require a phenomenon called super-symmetry. With super-symmetry, every fermion would have a boson twin, and vice-versa. TCP that behaves like boson should accompany its supersymmetrical partner TRP that functions like fermion in the generalized simpler way. Thus TCP like boson cannot have anti-particle. But TRP that functions like fermion should have its anti-particle and here it is shown as Anti-TRP (see Scheme-I). It is to be noted that these TCP and TRP function like wavicle: wave-particle duality.

1.2. In contrast to the usual linear sequence of matter, body, life, brain, mind, consciousness, here the proposed cyclic sequence is first universal consciousness (a 
functional state of the universal mind), and then matter, body, life, brain, and regeneration of mind and consciousness. The evolution of life with mind and consciousness is possible purely due to the inherent existence of universal consciousness which exists along with the universe. The human nervous system is evolved to provide an appropriate material structure to individualize the universal consciousness, a characteristic of reality, pervading all manifestations.

Consciousness model of Pal et al [1-3] involving TCP, TRP and thought force (TF) signifies the existence of universal consciousness that exists along with the universe. Pal et al [3] showed that this universal consciousness is a functional state of Universal Mind (UM). Pal et al [3] explained that the UM is evolved at the Big Bang from the eternal Void (see Scheme-I). This Void, in turn, is the source of infinite energy. And this UM is a finer matter. The individual mind being a constituent of the UM is also a finer matter. The constituents of the UM and individual mind are the same. The ultimate constituents of matter and mind are the same as both mind and matter are aspects of one fundamental reality, which is called UM. The brain is the mediating link or interface between the individual mind and body.

Pal et al [3] explained that the constituents of the UM are the ultimate constituents of matter itself as everything in this universe is a manifestation of this UM. Pal et al [3] expressed that the UM is constituted by these TCP and TRP in the inherent presence of thought force (TF). Pal et al [1, $3]$ further explained that the ultimate constituents of matter and mind are these TCP and TRP in the inherent presence of thought force (TF) in vitro and thought force (TF) in vivo [see Scheme-I]. Pal [5] explained the existence of the thought force (TF) that, in turn, is the primordial quantum field.

1.3. Pal et al [3] developed three different equations expressing the quantized energy ( $\left.\mathcal{E}_{T}\right)$ of TCP. The value of $\mathcal{E}_{T}$ in one of the three equations is shown below:

$$
\mathcal{E}_{T}=4.384 \times 10^{-16} \mathrm{erg} \equiv 2.73 \times 10^{-4} \mathrm{eV} \equiv 2.73^{0} \mathrm{~K} \cong C M B R \text { temperature } \equiv 2.725^{0} \mathrm{~K}
$$

It is to be noted that $1 \mathrm{erg} \cong 0.6241807 \times 10^{12} \mathrm{eV}$ and $10^{-4} \mathrm{eV \cong 1^{0 }} \mathrm{K}$ (where $\mathrm{K}=$ Kelvin) as indicated by Weisskopf [6].

1.4. Pal et al [3] expressed that the CMBR temperature is due to $\varepsilon_{T}$, the quantized energy of TCP where $\varepsilon_{T}=4.384 \times 10^{-16} \mathrm{erg} \equiv 2.73^{\circ} \mathrm{K}$ that is very close to $2.725^{\circ} \mathrm{K}$ (CMBR temperature). As per $\mathrm{Pal}$ et al [3], it is interesting to note that all of the three different equations ultimately give rise to the same result that is equivalent to the CMBR temperature. A sort of relationship is thus observed between the $\varepsilon_{T}$ and the CMBR temperature. This coincidence is thus signifying a probable role of TCP on the maintenance of CMBR temperature. Further, this coincidence is also signifying the existence of these TCP in the presence of TRP. TCP cannot exist without TRP and vice versa. The presence of TCP, TRP and Thought force (TF) in the universe is thus indicated and expressed mathematically. At present we are unable to explain when and how these TCP and TRP decoupled from the primordial cosmic soup.

Pal et al $[1,3]$ showed that the quantized energy $\left(\varepsilon_{T}\right)$ of TCP is responsible to cause the universal consciousness as well as the cosmic microwave background radiation temperature. The individual consciousness owes its origin to the universal consciousness created by the same $\varepsilon_{T}$.

Ultimately this $\boldsymbol{\varepsilon}_{T}$ represents universal consciousness. The existence of CMBR temperature indicates the existence of the TCP in the presence of TRP. The existence of TCP ensures the existence of the thought force (TF). Further,

this TF being an expression of the quantized energy $\left(\mathcal{E}_{T}\right)$ of TCP exerts its functions both in vitro and in vivo.

\section{Thought Force}

Pal [5] explained the existence of Thought force $\left(\mathrm{T}_{\mathrm{F}}\right)$. This Thought force $\left(\mathrm{T}_{\mathrm{F}}\right)$, an expression of the universal consciousness, is the primordial quantum field that, in turn, functions as the primary unified field. This $\mathrm{T}_{\mathrm{F}}$ being an expression of the universal consciousness is applicable to any inanimate object as well as to any biological system (having thinking ability). Thus the $\mathrm{T}_{\mathrm{F}}$ being an expression of the universal consciousness exerts its functions both in vitro and in vivo.

Physicists determined that underlying quantum fields give birth to elementary particles. Pal [5] expressed that the thought force $\left(\mathrm{T}_{\mathrm{F}}\right)$ is the primordial quantum field. Thought force $\left(T_{F}\right)$ being the primordial quantum field functions as the primary unified field. Thought force $\left(\mathrm{T}_{\mathrm{F}}\right)$ being the primordial quantum field gives birth to TRP that appears to be the origin of all the matter particles. TCP is the carrier of thought force $\left(\mathrm{T}_{\mathrm{F}}\right)$ that, in turn, appears to be the origin of all the fields. TCP thus appears to be the origin of all the field particles (see Scheme-I).

In a purpose to involve both the non-living and living systems of the world, Pal [5] has shown the existences of these TCP, TRP and thought force $\left(\mathrm{T}_{\mathrm{F}}\right)$ in vitro and thought force $\left(\mathrm{T}_{\mathrm{F}}\right)$ in vivo.

Pal (5) expressed that the non-living system of the world is governed by the thought force $\left(\mathrm{T}_{\mathrm{F}}\right)$ in vitro and this Thought force $\left(\mathrm{T}_{\mathrm{F}}\right)$ in vitro gives rise to $\mathrm{T}_{\mathrm{F}}$ (micro), SNF, EMF, WNF, GF and $\mathrm{T}_{\mathrm{F}}$ (macro)

where TF $($ micro) $=$ Thought force in microcosm, SNF = Strong nuclear force, $\mathrm{EMF}=$ Electromagnetic force, $\mathrm{WNF}=$ Weak nuclear force, GF = Gravitational force and TF (macro) $=$ Thought force in macrocosm. It is to be noted here that TF (micro) is a stronger force than the SNF and $\mathrm{TF}$ (macro) is a weaker force even than the GF. 
Pal (5) also expressed that the living system of the world is governed by the thought force (TF) in vivo and this Thought force (TF) in vivo is a type of force that represents the biological 'thought' which is the action of mind. This 'thought' being a type of force controls the 'thought processes' involving the firing of neurons through the quantum mechanical activities of these TCP and TRP in the presence of consciousness. This consciousness, in turn, is the quantized energy $\left(\varepsilon_{T}\right)$ of TCP. The thought force (TF) in vivo is demonstrated in numerous experiments in which thought has an effect on a physical process (often known as mind over matter). This biological 'thought' is a type of force that can cause movement. Controlling movement through thought alone is observed in several experiments conducted by many scientists as indicated by Pal (5). These experiments thus signify the existence of thought force (TF) in vivo.

Pal [5] explained the existence of TF (micro) (= Thought force in microcosm). This TF (micro) is the strongest interaction (a new class of 'extra strong' interaction). It is stronger than SNF (Strong Nuclear Force).

Pal [5] has also shown the existence of TF (macro) (= Thought force in macrocosm). It is the "weakest force" which is much weaker even than the gravity.

\section{How to Define and Interpret 'Time'?}

There is an arrow of time and there is something strange in the way time relates to consciousness. It is puzzling fact that the fundamental laws of both the quantum world as well as the large-scale universe are independent of time, and yet humans are actually aware of the arrow of time. Roger Penrose feels that this is a profound issue and this is the reason that both in classical physics and quantum physics there is no place for consciousness. In his book Shadows of the Mind Roger Penrose says, "Consciousness is a part of the universe". He believes that there is an objective world out there which exists whether it is perceived by a human mind or not, whose most remarkable manifestation, consciousness, is open to study by modern science. "Science will have to be quite seriously changed in order that consciousness can be accommodated". Penrose says, "We need something extended beyond that, where the idea of evolving time can actually make sense".

As per Hawking [7], there are at least three different arrows of time

(1) The cosmological arrow of time begins at the Big Bang, the beginning of the universe; because the earlier time before the Big Bang could not just be defined. This cosmological arrow of time is the direction of time in which the universe expands rather than contracts.

(2) The thermodynamic arrow of time is the direction of time in which the disorder or entropy increases.

(3) The psychological arrow of time is the direction of time in which we feel time passes: the direction in which we remember the past but not the future. The psychological arrow is essentially the same as the thermodynamic arrow because these two would always point in the same direction.

\subsection{What are the Characteristics of 'Time'?}

Regarding the characteristics of "time", the following points are to be taken into account as indicated by Hawking [7]:

(1) The theory of relativity reveals that "time" is not absolute. It can expand or contract; nothing can travel faster than the speed of light.

(2) Time begins at the 'Big Bang' singularity at which the universe is supposed to begin.

(3) Time would come to an end at the 'Big Crunch' singularity at which the universe is supposed to come to an end.

(4) Time also comes to an end at the singularity inside a black hole, which is formed due to gravitational collapse of a star to infinite density.

(5) There is a scope to use an idea of "imaginary time" (by incorporating $i=\sqrt{-1}$ ) through which the distinction between time and space disappears completely.

(6) There is no singularity or boundary in imaginary time, leading to the conclusion: The boundary condition of the universe is that it has no boundary. In imaginary time, the time is indistinguishable from directions in space.

(7) If one can utilize the 'quantum theory of gravity' (by unifying gravity with quantum mechanics) along with the idea of "imaginary time", then one would be able to open up a new possibility, in which there would be no boundary to space-time and there would be no singularity at which all the laws of science usually break down. This will lead to the totally "self-contained" universe ........The universe is neither created nor destroyed.....it is eternal.

(8) The arrow of time: The psychological arrow $=$ The thermodynamic arrow which agrees well with the cosmological arrow of time.

(9) "Time" is not completely separate from and independent of "space", but is combined with "space" to form what is called "space-time continuum".

Every event in this observable universe occurs on the platform of time through which such an event is generally being expressed. The concept of personal time (psychological time) begins to function to a person at the onset of the function of his or her conscious mind.

\section{Consciousness may be Defined as the 'SELF-ORGANIZED' Capability of any Living being to Activate TCP and TRP}

Pal et al [3] expressed that consciousness may be defined as the 'self-organized' capability of any living being to activate TCP and TRP, the ultimate constituents of mind 
and matter and to exert its functions. What can generate, maintain and activate TCP and TRP is called animate having active consciousness and what cannot is called inanimate, i.e., devoid of active consciousness. As anything inanimate does neither have the power to activate the TCP and TRP nor have the capability to catalyze the activity of TCP and TRP, so it cannot generate consciousness. On the contrary, anything animate has the 'self-organized' power to generate, activate and catalyze the activity of TCP and TRP in order to generate and maintain consciousness along with the vital living force indicated by $\mathrm{Pal}$ et al [1-3]. Prigogine et al [8] explained "Self-Organization in NonEquilibrium Systems". Prigogine [9] further explained "From Being To Becoming".

Consciousness in living organisms is a process which involves the quantum mechanical activities of these TCP and TRP, the ultimate constituents of any matter as well as any mind in the inherent presence of thought force $\left(\mathrm{T}_{\mathrm{F}}\right)$ in vitro and the thought force $\left(\mathrm{T}_{\mathrm{F}}\right)$ in vivo as indicated by $\mathrm{Pal}$ [5]. These TCP and TRP govern the activities of neurons (not the other way round). Neurons are simply the equipments used to generate consciousness and awareness.

The consciousness itself is functioning as an inter-linking agent between the animate and inanimate through the quantum mechanical activities of these TCP and TRP in the inherent presence of Thought force $\left(\mathrm{T}_{\mathrm{F}}\right)$ in vitro and Thought force $\left(\mathrm{T}_{\mathrm{F}}\right)$ in vivo as indicated by Pal et al [1-3].

\section{Space-Time}

Space and time are dynamic quantities and they not only affect but also are affected by everything that happens in the Universe. Hawking [7] mentioned, "Time is not completely separate from and independent of space but is combined with it to form what is called 'space-time continuum'. Space can not exist without time and vice versa.

Pal [10] explained that time without consciousness is not possible. But consciousness without time is possible. Time of any kind cannot exist without the existence of consciousness. Thus Space-time without consciousness is not possible. Space-time exists only in the existence of consciousness. Although space-time continuum is thought to be a four-dimensional object, yet Space and Time appear to be subjective in nature.

\subsection{Are Space and Time Subjective?}

Bhaumik [4] mentioned: Wheeler has speculated that reality is created by observers in the universe. His timebending notion is that our "observership brings the universe into being".

Wheeler indicated that the process of recognizing an observable object is really a type of participation on the part of the observer for a specific object to be observed. This process of recognizing an observable object is possible through the projection of mind of the observer in the presence of his / her consciousness.

One of the central notions of human existence has been that of a Being prior to space, time, and substance. The world described by science must be a picture of mindindependent reality not as it is seen through the selective and deforming lens of our own sensory and mental structures through the inexact filter of consciousness. It seems we are forced to acknowledge that we cannot know mind-independent reality as it is.

In Einstein's world of relativity, space and time are often described as observer-dependent, and thus subjective. Then, is Relativity Subjective? Some commentators suggest that Einstein's theory is more malleable than old-fashioned Newtonian physics. Relativity seems less rigid. Space and time seem to blend in a kind of blur.

Haught [11] writes: Relativity physics does construe the cosmos in a way that allows for a more intimate indwelling of both mind and God in nature. . . The physical universe is not easily separable from the observer's mind, a feature we locate at the heart of personality. We cannot extricate our minds from nature in such a way as to have an absolute perspective on things. We are now becoming convinced that mind is much more deeply embedded in the physical universe than we had for centuries suspected. All measurements turn out to be subjective-dependent on the motion of the observer.

Gilkey [12] pointed it out, "The primary qualities of classical physics . . . mass, velocity, space-time distance---are gone---all is observer related".

Casti [13] expressed, "There is no such thing as an objective, observer-independent event, at least insofar as describing its location in space and time". The relationship between mind and nature is essentially the same in the 20th century and in the 17 th.

\subsection{Are Space and Time Real? And what is Reality?}

There are three kinds of space: (1) abstract geometrical concepts; (2) the space of science and ordinary experience; and (3) real space (not absolute!) which science approximates.

What is space? That is pure, empty space? Or what is time in itself? In discussions of relativity, people incessantly talk about measuring sticks and clocks. We can use a yardstick to measure a thing, but how can we measure empty space? There's nothing there to measure. A clock can measure an event, but how do we measure pure time? By what standard do we check our yardsticks and clocks? Space is where we put things. Time is when events happen. Our instincts about space and time are quite valid. We can't touch pure, empty space, but it is waiting there-room for us to put something.

There is a science of space and time, which utilizes operational definitions. A straight line is the shortest distance between two points. Another principle in the science of space and time is that a light ray follows a straight line. Science is an attempt to describe reality. Scientists have discovered that Riemannian geometry is the best representative of the world we live in. Also, science uses measuring devices to measure the things we find in this world. How can we be sure that there is such a thing as "real space"? In the world, how can empty space be real? We can't touch it. The real world includes non-tangible 
elements. Suppose we have an object, and we have some empty space where we would like to place the object. Will it fit? Well, first we measure the object. Then we measure the empty space-that is, we measure the physical surroundings that define the extent of the empty space. Then we decide. Empty space has some remarkable properties. It is available to support the propagation of electromagnetic waves. We can use a light ray to explore empty space, because light always travels in a "straight" line in a vacuum.

\subsection{Characteristics of Space-Time}

As per the ancient Vedanta, there is only one Infinite Existence called BRAHMAN. This BRAHMAN is more or less equivalent to the Void of the modern science. This Void is not the meaning of nothingness. This Void is the source of infinite energy. Anything and everything of this universe are its manifestations. Hawking [7] mentioned, "The Heisenberg's uncertainty principle established that the Void is filled with infinite pairs of virtual particles and antiparticles. These pairs would have an infinite amount of energy and therefore, by Einstein's equation: $\mathrm{E}=\mathrm{mc}^{2}$, they would have an infinite amount of mass". Without the uncertainty principle to forbid nothingness, there might not even be a universe.

The nature of time's arrow has been discussed by physicists, philosophers, sages and charlatans but there is no agreement. The world of atomic particles shows us that there is really no time's arrow at the quantum level. "Time" itself loses its meaning, and at the Planck scale the words "before" and "after" are without application as indicated by Wheeler [14]. [Planck length = L $\left.\left(h G / c^{3}\right)^{1 / 2}=4.04 \times 10^{-33} \mathrm{~cm}\right]$.

Wheeler [14] pointed out, "Three-dimensional geometry carries information about time (Wheeler et al [15]). The geometry is not deterministic, even though it looks so at the every day scale of observation. Instead, at microscopic [or Planck length $\left.=\mathrm{L}=\left(h G / c^{3}\right)^{1 / 2}=4.04 \times 10^{-33} \mathrm{~cm}\right]$ scale, it "resonates" between one configuration and another and another. The ${ }^{(3)} G^{\prime} s$ with appreciable amplitude are too numerous to be accommodated into any one space-time. Thus the uncertainty principle declares that space-time is only an approximate and classical concept. In reality there is no such thing as space-time. "Time" itself loses its meaning, and [at the Planck scale] the words "before" and "after" are without application".

This is due to the fact that approximately $10^{15}$ times Planck length $\left(\approx 4.04 \times 10^{-33} \mathrm{~cm}\right)$ are required to arrive at the radius $\left(=8.28 \times 10^{-18} \mathrm{~cm}\right)$ of a TCP. It is to be noted that the theoretically calculated radius of a TCP in the inherent presence of TRP within a nucleon = $8.28 \times 10^{-18} \mathrm{~cm}$. It has already been mentioned that the quantized energy $\left(\varepsilon_{T}\right)$ of TCP is responsible to cause the generation of consciousness. And further there is no manifestation of 'time' unless there is the generation of consciousness $=\varepsilon_{T}=m_{T} c^{2}=h c / \lambda_{T}$ (where $m_{T}=$ quantized mass of the TCP, $\mathrm{c}=$ free- space velocity of light, $\mathrm{h}=$ Planck's quantum constant, $\lambda_{T}=$ wavelength of the TCP).

The manifestation of any type of "time" is solely dependent on the manifestation of consciousness $\left(=\varepsilon_{T}\right)$. The concept of personal time (psychological time) begins to a person at the onset of the function of his or her "conscious mind" only. 'Time' is bound to the 'mind'. Even the existence of cosmological arrow of time is also dependent on the existence of universal consciousness that, in turn, is the quantized energy ( $\varepsilon_{T}$ ) of TCP. This $\varepsilon_{T}$ represents universal consciousness.

Consciousness is a functional state of mind. Presence of consciousness signifies the presence of mind and vice versa. It is to be understood that consciousness is the realization of existence as indicated by Pal et al [2]. There is another term called unconscious state. The most usual unconscious state is sleep. A deeper form of unconsciousness is called a coma.

As 'time' is solely dependent on the manifestation of consciousness $\left(=\mathcal{E}_{T}\right)$, so time is absent to an unconscious person. Thus time is absent to a sleeping person. Similarly, time is absent to a person in coma state.

\section{The Gravity is Related with the Quantized Energy $\left(\varepsilon_{T}\right)$ of TCP}

The gravity is related with the quantized energy $\left(\varepsilon_{T}\right)$ of TCP through the following expression:

$$
G_{F}=\frac{1}{K}\left(\varepsilon_{T} / R\right)^{2}=G / c^{4}\left(\varepsilon_{T} / R\right)^{2}=\left(G / c^{4}\right)\left[T_{F}(\text { macro })\right]^{2}=\left(G / R^{2}\right)\left(\varepsilon_{T} / c^{2}\right)^{2} \ldots
$$

Where

$$
\begin{aligned}
& G_{F}=\text { gravitational force, } \\
& \mathrm{K}=c^{4} / G, \\
& \mathrm{c}=\text { free-space velocity of light, } \\
& \mathrm{G}=\text { Newton's gravitational constant, } \\
& \mathcal{E}_{T}=\text { quantized energy of the TCP radiated from the } \\
& \text { radiant mass of the universe, }
\end{aligned}
$$

$$
\begin{aligned}
& \mathrm{R}=\text { radius of the universe }=1.482 \times 10^{29} \mathrm{~cm}, \\
& c / H_{0}=\mathrm{L}(\text { Hubble length })=\text { radius of the universe }=\mathrm{R} \\
& =1.482 \times 10^{29} \mathrm{~cm}, \\
& H_{0}=\text { present value of Hubble's parameter }= \\
& 2.023988 \times 10^{-19} \mathrm{sec}^{-1} .
\end{aligned}
$$

$\mathrm{TF}($ macro $)=$ Thought force in macrocosm $=\varepsilon_{T} / \mathrm{R}$. 
The gravity is thus related with the quantized energy $\left(\varepsilon_{T}\right)$ of TCP radiated from the radiant mass of the universe. Thus TCP or TRP once detected may shed immense light on the 'gravity' (even on 'black holes') in order to detect 'gravity waves', ripples in 'space-time' predicted by Einstein. Rovelli [16] expressed, "Through his "Ashtekar loop" for quantum dynamics of geometry of space Ashtekar wants to say, "Einstein showed gravity is nothing but the geometrical manipulations of space-time. Matter curves the space around it, and space tells matter how to roam in that curved space. So like matter, space, too, is very much dynamic. There is no spectator in cosmic dance, everyone is an actor in the big show."

Further, $\mathcal{E}_{T}$ is found to be directly proportional to $\mathrm{c}$ where the proportionality constant, $\mathrm{K}=\mathrm{h} / \boldsymbol{\lambda}_{\mathrm{T}}$. The possibility of the existence of a universal web of "quantum fields" also signifies the existence of the $\mathrm{T}_{\mathrm{F}}$ (micro) and $\mathrm{T}_{\mathrm{F}}$ (macro) due to the existence of quantum mechanical activities of the TCP in the presence of TRP.

\section{Does the Time Create Energy or Vice- Versa? Is there any Relationship between the Time and Energy?}

The uncertainty principle dictates that the energy in a field or in 'empty space' is not constant, but can fluctuate more and more wildly the smaller the period of time that one looks at it. Such quantum fluctuations during the Big Bang are now thought to be the origin of galaxies. In some theories, the universe itself is a quantum effect, the result of a fluctuation in some sort of pre-universal nothingness. Without quantum fluctuations, there would be no galaxies, stars or friendly warm planets. Without the uncertainty principle to forbid nothingness, there might not even be a universe.

The time, being bound to the mind, is forming a bridge between the internal world (mental world) and the external world (physical world) manifesting its presence through the function of conscious mind via the quantum mechanical activities of these TCP and TRP, the ultimate constituents of matter and mind in the inherent presence of thought force $\left(\mathrm{T}_{\mathrm{F}}\right)$ in vitro and thought force $\left(\mathrm{T}_{\mathrm{F}}\right)$ in vivo. So there is always a bridge between the internal world and external world through the quantum mechanical activities of TCP and TRP projected by the mind in the presence of consciousness. This consciousness is responsible for the generation of time as well as space called space-time continuum. Time is frequently elaborated philosophically as a peculiar form of energy by expressing it in the following language: "Time is a great healer". Is the time itself then a peculiar form of energy? Does the time create energy? Or does the energy generate time?

Pal [10] explained that time without consciousness is not possible. But consciousness without time is possible. Time of any kind cannot exist without the existence of consciousness. Pal et al [3] showed that the quantized energy $\left(\varepsilon_{T}\right)$ of TCP represents universal consciousness.
The individual consciousness owes its origin to the universal consciousness created by the same $\varepsilon_{T}$. This universal consciousness functions as a typical form of energy like light. Consciousness may thus be defined as a mental light through which a blind person can recognize any object through the use of his / her mental light.

Time does not create energy but consciousness being a typical form of energy (like light) generates the concept of time (and space). Time owes its origin to consciousness (a typical form of energy like light) and this consciousness is a functional state of mind to which the time is bound. Similarly the universal consciousness is a functional state of the universal mind to which the cosmological arrow of time is bound.

In order to detect the relationship between the time and energy, it is found that there is an inverse relationship between the time and the "intensity of the field of energy" as it is evident by the dimensional analysis of Einstein's equation: $\mathrm{E}=\mathrm{mc}^{2}$. If it is a fact that there is an inverse relationship between the energy and time, then when time becomes zero at the inside singularity of a black hole (formed due to the gravitational collapse of a star), will the energy inside the black hole really be infinity? Time is supposed to come to an end at the inside singularity of a formed black hole. What is the fate of 'energy' inside the black hole? By understanding the magnitude, character, and function of energy inside the black hole, the interrelationship between the time and energy can be properly detected, characterized and established.

According to the Einstein's theory of relativity, there is no unique absolute time, but instead, each individual has his own personal measure of time that depends on where he is and how he is moving. It is therefore concluded that time can expand or contract as it is expressed by the world famous "Twins paradox" which runs like this: Keeping one of the twins on the earth, if the other fellow goes for a trip in a space-ship at nearly the speed of light, then, after returning from his space trip, he would see that he is much younger than the one who was staying on the earth. The explanation that is usually given for this expansion or contraction of time, as expressed by Hawking, is that the time should run slower near a massive body like earth. This is due to the fact that when light travels upwards crossing earth's gravitational field, it looses energy and thus its frequency goes down, as the energy of light is directly proportional to its frequency. It would thus appear to someone high up that everything down below is consuming longer time to happen. Among the surprising effects of relativity are that a moving clock runs slower than a clock at rest, and that time in a mountain top runs faster than time at sea level, because gravity is stronger at sea level and gravity slows time down. This phenomenon leads to the "Twins paradox" if one has the idea of absolute time at the back of one's mind as it is mentioned by Hawking [7].

All the parameters on the right hand side of the Equation (1) are more or less physical constants. Even $\mathrm{T}_{\mathrm{F}}$ (macro) itself is a type of universal constant. Accepting $\mathrm{T}_{\mathrm{F}}$ (macro) to be a universal constant, the usual dimensional analysis of the Equation (1) would signify that 


\section{GF $\alpha$ Time $^{4}$}

The Equation (2) indicates that the physical time would have to expand with the increase in $G_{F}$ and similarly, the physical time would contract with the decrease in $\mathrm{G}_{\mathrm{F}}$.

The concept of psychological time would thus have to expand to a person existing within the field of lower energy on the surface of earth where there is a gravitational field, which has negative energy. Can this prediction of general relativity regarding the expansion of time near a massive body be explained by the fact that the time would have to expand to a person existing within a field of lower energy level relative to the other fellow who is existing (in the space-ship) within a field of higher energy level? This contraction and expansion of time indicate the presence of an inverse relationship between the time and "intensity of the field of energy" where one is staying and how one is moving.

It is further to be noted that the uncertainty relationship between the time and energy is given as

$$
\Delta \mathrm{t} \Delta \mathrm{E} \approx \overline{\mathrm{h}}=\mathrm{h} / 2 \pi
$$

where $\mathrm{h}=$ Planck's Quantum constant and $\overline{\mathrm{h}}=$ Reduced Planck constant.

The relationship indicates that the uncertainty of the energy of the particle is dependent on the time interval used, since the particle must be observed for a time $\Delta t$ to be certain that the particle has passed the point of observation. If an energy measurement is performed in a time interval $\Delta t$, there would be a corresponding minimum uncertainty in the energy given by the above equation.

\section{TCP, TRP and Thought Force $\left(T_{F}\right)$ Play Significant Roles in the Evolution of Time}

Pal [10] explained that TCP, TRP and Thought Force (TF) play significant roles in the evolution of time.

These TCP, TRP and thought force (TF) in vitro and (TF) in vivo play their significant roles both in the non-living and living systems of this universe, although it is hard at present to prove their physical existence thoroughly except the maintenance of CMBR temperature and the existence of thought force (TF) in vivo. The controlling movement through thought alone is observed in several experiments conducted by Birbaumer [17], Donoghue et al [18-21], Hatsopoulos [22] and Miguel [23]. These experiments thus signify the positive proof for existence of the TCP, TRP and thought force (TF) in vivo. The existence of the thought force (TF) in vivo confirms the presence of $\mathcal{E}_{T}$. This $\mathcal{E}_{T}$ is the quantized energy of TCP radiated by the radiant mass of this universe.

Pal et al [3] indicated that these TCP and TRP are the ultimate constituents of any matter as well as any mind in the inherent presence of the thought force $\left(\mathrm{T}_{\mathrm{F}}\right)$ in vitro and thought force $\left(\mathrm{T}_{\mathrm{F}}\right)$ in vivo.

Pal et al $[1,3]$ explained that the eternal 'Void' has been covered by the mind to function as the Soul of individual

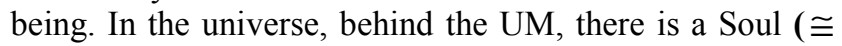
VOID). In the individual, behind the individual mind

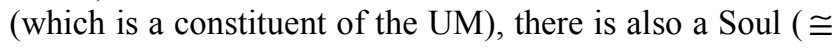

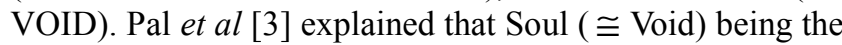
master ruler of the body exerts its functions through the finer instrument called mind. The mind (with the help of TCP and TRP) exerts its functions through the brain and spinal cord (CNS) along with the other parts of the nervous system (PNS along with ANS) in the biological systems in order to grasp this physical universe, of course, in the inherent presence of consciousness. This consciousness itself is acting as an inter-linking agent between the animate and inanimate through the quantum mechanical activities of these TCP and TRP. Thus these TCP and TRP being the ultimate constituents of mind as well as matter play the most significant roles in many present day scientific enigmas.

As per Edelman, a Nobel Laureate, consciousness is a process, a chain of events, rather than a 'thing' contained in a special part of the brain. Pal et al $[1,3]$ expressed that consciousness is a process involving the quantum mechanical activities of these TCP and TRP that are the ultimate constituents of any matter as well as any individual mind in the inherent presence of thought force $\left(\mathrm{T}_{\mathrm{F}}\right)$ in vitro and thought force $\left(\mathrm{T}_{\mathrm{F}}\right)$ in vivo.

It is our plausible opinion that the manifestation of time is simply and solely dependent on the generation of consciousness that is the quantized energy $\left(\varepsilon_{T}\right)$ of TCP.

Lynds [24] says, "It should become clear that no matter how small the time interval, or how slowly an object moves during that interval, it is still in motion and its position is constantly changing, so it can't have a determined relative position at any time. Indeed, if it did, it couldn't be in motion. Thus all the motion paradoxes stand as mere mathematical tricks and can be explained in my theory. It is something entirely subjective that we project onto the world around us. That is, it is the outcome of brain function and consciousness".

In our proposition, the manifestation of time is absolutely dependent on the generation of consciousness (= $\left.\mathcal{E}_{T}\right)$. That is why Lynds [24] has to point out that time is the outcome of brain function and consciousness [that is the quantized energy $\left(\mathcal{E}_{T}\right)$ of TCP].

Georgiev [25] expressed, "Observed rare cases in the clinical practice of patients with time agnosia clearly show that consciousness is possible without perception of time and that for these patients apparent dynamics of observed objects do not produce feeling of "time flow", as intuitively expected. Thus if our mind operates at $100 \mathrm{GHz}$ (dynamical timescale of 10 picoseconds), every single "occasion of consciousness" experienced as "present" could be result from $10^{10}-10^{11}$ conscious steps sharing the same time label". He further pointed out, "Recently proposed models of boson condensation as mind process (with $10^{-14} \mathrm{~S}$ dynamics) cannot provide any biological function taking part at this timescale that is why I think the $100 \mathrm{GHz}$ 
conscious informational processing is the fastest possible".

\section{Time without Consciousness is not Possible. But Consciousness without Time is Possible}

Pal [10] explained that time without consciousness is not possible. But consciousness without time is possible.

As per Georgiev [25], our mind operates at $100 \mathrm{GHz}$ (dynamical timescale of 10 picoseconds) where $100 \mathrm{GHz}=$ $10^{11} \mathrm{~Hz}$ and 10 picoseconds $=10^{-11}$ sec. It can be shown that the calculated frequency of TCP $=0.6612 \times 10^{11} \mathrm{~Hz}$ that agrees well with $100 \mathrm{GHz}\left(=10^{11} \mathrm{~Hz}\right)$. And the calculated time period of the TCP $=1.51 \times 10^{-11} \mathrm{sec}$ that is equivalent to 10 picoseconds $=10^{-11}$ sec. It is thus apparent that there is a remarkable relationship between the operational mind and the quantum mechanical activities of these TCP and TRP with which every mind is assumed to be constituted. Thus our assumption that mind is constituted and operated by these TCP and TRP is found to be correct through these propositions and experimental observations. These TCP and TRP can thus form a possible bridge between mind and matter in the presence of thought force $\left(\mathrm{T}_{\mathrm{F}}\right)$ in vitro and thought force $\left(\mathrm{T}_{\mathrm{F}}\right)$ in vivo.

At the onset of the Big Bang, the time as well as space is generated along with the universal consciousness that, in turn, is a functional state of the Universal Mind (UM). This UM is shown here to be constituted by these TCP and TRP in the inherent presence of thought force $\left(\mathrm{T}_{\mathrm{F}}\right)$, an expression of the universal consciousness that, in turn, is the quantized energy $\left(\varepsilon_{T}\right)$ of TCP.

These TCP and TRP play the fundamental roles for the initiation of consciousness $\left(=\varepsilon_{T}=m_{T} c^{2}\right)$ through which the evolution of light (at the Big Bang) and its velocity (c) can be realized. Thus, the apparent origin of the physical time appears to be the origin of evolution of light and its evolved velocity (c) at that point of the beginning of the universe.

Light travels fast and far, but even the light stops at a black hole. A black hole is a star that is so massive that it collapses to a practically no volume under its own gravity. The gravitational force in a black hole is so intense that even light cannot escape from it. It is to be noted that time (as well as space) becomes zero at the inside singularity of a black hole. The origin of the physical time thus appears to be related with the origin of the evolution of light and its evolved velocity (c). Thus, the velocity (c) of light as well as time is always related with consciousness = $\varepsilon_{T}=m_{T} c^{2}=h c / \lambda_{T}$. And the consciousness is thus conjectured here to be the 'mental light'. It is to be noted that $\mathcal{E}_{T}$ is found to be directly proportional to c (free-space velocity of light) where the proportionality constant, $K=h / \lambda_{T}$ (where $\mathrm{h}$ is Planck's quantum constant =
$6.63 \times 10^{-27} \mathrm{erg} . \mathrm{sec}$, and $\lambda_{T}$ is the wavelength in $\mathrm{cm}$ of the TCP).

Pal et al [2] explained, "Consciousness is the realization of existence, and there are as many states of consciousness as there are states of existence. Every living being has a consciousness of its own depending on complexity of the brain and the activity of the viable numbers of TCP and TRP; and the state of its consciousness changes every moment of time. Consciousness is the perception of the relation it bears to things and as this relation changes, consciousness changes its character. Consciousness itself does not change; it only moves up and down on the 'scale of the realization' of existence through the 'sub-conscious', 'conscious' and 'super-conscious' states".

Time of any kind cannot exist without the existence of consciousness. Time without consciousness is not possible. But consciousness without time is possible. That is why Georgiev [25] pointed out, "Consciousness is possible without perception of time." It is really very difficult to understand the fact that consciousness alone can exist independently without perception of time. Consciousness can exist independently in the form of super-consciousness, a state that is usually enjoyed and experienced by a real 'YOGI' (who practises meditation). In the super-conscious state, the perception of time becomes zero. It also indirectly signifies the existence of consciousness prior to space, time, and substance. It thus indicates the existence of universal consciousness that, in turn, exists along with the universe, thereby, proving the fact that consciousness, not matter, is the ground of all existence.

Consciousness, not matter, is the ground of all existence, declares University of Oregon physicist Goswami through his published (1993) book, "The Self-Aware Universe: How Consciousness Creates the Material World". He accepts the Vedantic view and holds that the universe is self-aware, and that consciousness creates the physical world. Matter is an expression of mind, not separate from mind, but mind manifested materially.

\section{Probable Characteristics of Super- Consciousness}

The manifestation of any type of "time" is solely dependent on the manifestation of consciousness $\left(=\mathcal{E}_{T}\right)$. The concept of personal time (psychological time) begins to a person at the onset of the function of his or her conscious mind only. Time is bound to the mind. Even the existence of cosmological arrow of time is also dependent on the existence of universal consciousness that, in turn, is the quantized energy ( $\boldsymbol{\varepsilon}_{T}$ ) of TCP.

It is evident that when we remain vigorously absorbed in some kind of deep thought or when we remain in the exulted ecstatic mood (thereby existing within the high energy level mentally and internally) being vigorously involved with the beloved persons, then we feel that the time runs very quickly. In other words, the personal concept of time is contracted as the intensity of the field of energy 
is increased. On the other hand, the time appears to run very slowly (that is the time is expanded) when we fall in the distressed and hopeless condition in which we remain within the exhausted energy level mentally and internally. Thus whenever we remain in the state of high concentration of mind, thereby existing within a high energy level mentally and internally, we usually forget the existence of time. Time is contracted ultimately to zero. We then forget the existence of time altogether, although we are not really conscious about the intrinsic meaning of this vanishing of time and re-appearing of time.

The network of time, space and causation gives rise to the 'Name and Form' called Mâyâ by the Vedanta as expressed by Vivekananda [26]. Mâyâ is a simple statement of facts of the universe, of how it is going on; what we are and what we see around us, although the oldest idea of Mâyâ was the sense of delusion. The world is an illusion due to Mâyâ. No one can go out of one's self. No man can go beyond the limits that have been put upon him by the laws of time, space and causation, the network of which is called Mâyâ. The vanishing of time means the vanishing of one of the constituents of Mâyâ. This Mâyâ itself, in turn, is responsible for the manifestation of this relative world. If one at all likes to go beyond this relative world of Mâyâ in order to arrive at the absolute called "BRAHMAN" (@Void), then one would have to adopt the method of meditation for concentrating the mind (to deactivate or control the quantum mechanical activities of these TCP and TRP) in order to achieve the state of "superconsciousness": SAMADHI. At the super-conscious state the Yogi (Meditator) unifies himself with the universal soul

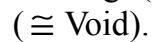

Pal et al [3] explained that the eternal Void has been covered by the mind to function as the Soul of the individual being. In the universe, behind the UM, there is a Soul ( $\cong$ VOID). In the individual, behind the individual mind (which is a constituent of the UM), there is also a Soul ( $\cong$ VOID)

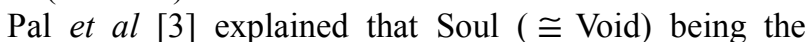
master ruler of the body exerts its functions through the finer instrument called mind. The mind (with the help of TCP and TRP) exerts its functions through the brain and spinal cord (CNS) along with the other parts of the nervous system (PNS along with ANS) in the biological systems in order to grasp this physical universe, of course, in the inherent presence of consciousness. This consciousness itself is acting as an inter-linking agent between the animate and inanimate through the quantum mechanical activities of these TCP and TRP. Consciousness is defined here as the 'self-organized' capability of any living being to activate TCP and TRP, the ultimate constituents of mind and matter. This consciousness, in turn, is the quantized energy $\left(\varepsilon_{T}\right)$ of TCP.

$$
L(\text { Hubble length })=R=c / H_{0}=\varepsilon_{T} \lambda_{T} / 2 E_{\min }=1.482 \times 10^{29} \mathrm{~cm}
$$

where

$$
\begin{aligned}
& \mathrm{R}=\text { radius of the universe } \\
& \mathrm{c}=\text { free-space velocity of light }=3 \times 10^{10} \mathrm{~cm} / \mathrm{sec},
\end{aligned}
$$

$H_{0}=$ present value of Hubble's parameter = $2.023988 \times 10^{-19} \mathrm{sec}^{-1}$,
Any matter as well as the individual mind is constituted by these TCP and TRP that, in turn, are originated from the same eternal Void. Thus the individual mind is operated by these TCP and TRP that, in turn, are ultimately governed by the same eternal Void. The individual mind being a constituent of the UM has covered the eternal Void to serve the role of individual Soul. This eternal Void is thus the common source and the source is still with us indicated by Bhaumik [4]. This Void serves the role of Soul being covered by the mind.

The mind-brain and brain-body links which are addressed by psychology, cognitive science, neuroscience and neurophysiology are ultimately governed by the quantum mechanical activities of these TCP and TRP in the inherent presence of thought force $\left(\mathrm{T}_{\mathrm{F}}\right)$ in vitro and thought force $\left(\mathrm{T}_{\mathrm{F}}\right)$ in vivo. Consciousness is a functional state of mind.

Pal et al [2] expressed that consciousness is the realization of existence, and there are as many states of consciousness as there are states of existence. Every living being has a consciousness of its own depending on complexity of the brain and the quantum mechanical activities of the viable numbers of TCP and TRP; and the state of its consciousness changes every moment of time. Consciousness is the perception of the relation it bears to things and as this relation changes, consciousness changes its character. Consciousness itself does not change; it only moves up and down on the 'scale of the realization' of existence through the 'sub-conscious', 'conscious' and 'super-conscious' states.

The super-conscious state is as good as the void ( $\cong$ soul) that is a source of infinite energy. It is obvious that although the super-consciousness and unconsciousness, the two extreme states of consciousness, apparently appear to be the same in nature, yet there is really a heaven and hell difference. In the super-conscious state the quantum mechanical activities of TCP and TRP are absolutely controlled for which there is an apparent absence of functional mind leading to the absence of any sign of 'life' as it is exhibited by any real 'YOGI' (meditator) who can 'recharge' himself to the 'life' function again as per his own desire.

Consciousness is a type of mental light. Superconsciousness is the purest form of mental light. The purest form of light would be the light that would be coherent, monochromatic and collimated.

LASER (Light Amplification by Stimulated Emission of Radiation) is a form of light. A beam of light, with exactly one frequency and one polarization and one direction for all of the photons is a laser beam. Super-consciousness may be thought to be a typical form of light like laser.

It can be shown that 
$\boldsymbol{\varepsilon}_{T}=$ quantized energy of the TCP radiated from the radiant mass

$$
\left(=1.16025 \times 10^{53} \mathrm{~g}\right) \text { of the universe = }
$$
$4.95 \times 10^{-16} \mathrm{erg}$,

$\lambda_{T}=$ wavelength of the TCP $=h c / \varepsilon_{T}=0.4018 \mathrm{~cm}$, $E_{\min }=$ minimum quantized energy = $h H_{0} / 2=6.70952 \times 10^{-46} \mathrm{erg}$.

It is known that

$$
\begin{gathered}
\lambda_{T}=h c / \varepsilon_{T} \\
m_{T}=\varepsilon_{T} / c^{2} \\
\tau_{T}=\lambda_{T} / c
\end{gathered}
$$

It is clear from the Equations (5), (6) and (7) that When

$$
\varepsilon_{T}=2 E_{\min }=13.41904 \times 10^{-46} \mathrm{erg}, \text { then }
$$

$\lambda_{T}=$ wavelength of the TCP $=\mathrm{R}=$ radius of the universe $=1.482 \times 10^{29} \mathrm{~cm}$,

$m_{T}=$ quantized mass of the TCP = $\varepsilon_{T} / c^{2}=1.4910044 \times 10^{-66} g$,

$v_{T}=$ frequency of the
$\varepsilon_{T} / h=2.0239879 \times 10^{-19} \operatorname{cps}\left(=H_{0}\right)$,

$\tau_{T}=$ time period of this $\mathrm{TCP}=\lambda_{T} / c=$ $h / \varepsilon_{T}=4.94 \times 10^{18} \mathrm{sec}=1.5665 \times 10^{11}$ years

$=$ theoretically calculated age of the universe.

Thus it is evident that in this case

$\mathrm{m}=$ mass of the source from which this TCP ( of $\lambda_{T}=R$ ) is radiated $=$

$$
h V_{p r} / \lambda_{T}{ }^{4} c=\varepsilon_{T}{ }^{4} V_{p r} / h^{3} c^{5}=6.25 \times 10^{-66} g
$$

where $\quad V_{p r}=$ present volume of the universe $=$ $(4 / 3) \pi\left(c / H_{0}\right)^{3}=2.365 \times 10^{88} \mathrm{~cm}^{3}$.

The 'super-conscious' state can be achieved by the way of meditation through the absolute control (i.e. deactivation) of TCP and TRP when $\varepsilon_{T}$ is likely to become $2 E_{\min }=13.41904 \times 10^{-46} \mathrm{erg}$ for giving rise to a value of $\lambda_{T}=R=$ radius of the universe $=1.482 \times 10^{29} \mathrm{~cm}, v_{T}=$ frequency of the TCP $=$ $\varepsilon_{T} / h=2.0239879 \times 10^{-19} \operatorname{cps}\left(=H_{0}\right)$ and $\tau_{T}=$ time period of this $\mathrm{TCP}=\lambda_{T} / c=h / \varepsilon_{T}=4.94 \times 10^{18} \mathrm{sec}=$ $1.5665 \times 10^{11}$ years $=$ theoretically calculated age of the universe. Average age of the universe indicated by Gamow $[27]=1.7 \times 10^{9}$ years .
Based on the best available measurements as of $2010^{\text {[update] }}$, the original state of the universe existed around 13.7 billion years ago as indicated by Komatsu [28] and Menegoni [29]. This time of 13.7 billion years is often referred to as the time when the Big Bang occurred as detected by CERN $[30 ; 2010]$ and indicated by Keohane [31;2010].

It is thus found that the age of the universe $=13.7$ billion years $\cong 0.137 \times 10^{11}$ years. Theoretically calculated age of the universe $=\tau_{T}=1.5665 \times 10^{11}$ years. This theoretically calculated age of the universe is about 11 times more than the recent observed value.

Could there be another type of black hole in our modern universe that was created before the Big Bang? Carr and Coley [32] have published a paper on arXiv, where they suggest that some so-called primordial black holes might have been created in the Big Crunch that came before the Big Bang, which supports the theory that the Big Bang was not a single event, but one that occurs over and over again as the Universe crunches down to a single point, then blows up again, over and over. This theory also indicates that we have an eternal, cyclical cosmos as expressed by Gurzadyan and Penrose [33], thus signifying the existence of eternal KALPA (Cycle) of the Vedanta as it is explained by Vivekananda [34].

\section{Discussion and Conclusion}

The intergalactic universal communication system is possible to be developed which cannot be attained by any conventional communication system. At the superconscious state, one can abolish not only the existence of time but also the quantum mechanical activities of these TCP and TRP, the ultimate constituents of matter and mind as it is indicated by $\mathrm{Pal}$ et al [3]. The radiating source of this particular TCP (of $\lambda_{T}=R$ ) at the super-conscious state is found to be $6.25 \times 10^{-66} \mathrm{~g}$. Although it appears to be impossible, yet it is found theoretically that the mass of a meditator (YOGI) in the super-conscious state is thoroughly and absolutely reduced (to emit this type of TCP) in order to generate what is called 'light of wisdom'. This super-conscious state is to be achieved for going beyond this relative world of Mâyâ (: the network of time, space and causation) without moving physically elsewhere outside this world. This state is called liberation (MOKSHA). This liberation means coming out of this 'bondage of mind' to the original void by controlling the quantum mechanical activities of these TCP and TRP and thereby, releasing them wishfully. This liberation means becoming the original void which, in turn, is the source of infinite energy and thus capable of creating anything and everything of this universe. That is why the learned persons in the field of 'philosophy, religion and spirituality' say, "We cannot get God, but we would have to become God". This is possible by the way of meditation for achieving liberation through the control of these TCP and TRP. He who has developed the power (through the meditation) to 
control these TCP and TRP can govern the universe; because he then becomes the 'free-man', the 'god-man'. Thus a meditator (YOGI) in the super-conscious state can arrive at the original void by controlling the quantum mechanical activities of these TCP and TRP and he is capable of releasing them wishfully as per his own desire to control anything and everything of the universe.

Here we like to point out what Wheeler [35] expressed, "Thomas Mann in his essay on Freud utters what Niels Bohr would surely have called a great truth ("A great truth is a truth whose opposite is also a great truth.") when he says, "Science never makes an advance until philosophy authorizes and encourages it to do so".

\section{References}

[1] D. Pal and A.U. De, Physics of consciousness and its model may provide guidelines to solve many scientific problems. Neuroquantology 1, 17-28 (2004)

[2] [2] D. Pal, and A.U. De, Consciousness model: Significance of thought-carrying particles and thought-retaining particles in quantum measurement as well as cognitive problem. NeuroQuantology 2, 115-116 (2005)

[3] D. Pal, and A.U. De, The cosmic microwave background radiation temperature signifying the existence of the thought-carrying particle, thought retaining particle and thought force. NeuroQuantology 10: Issue3; 428-442 (September 2012)

[4] M. Bhaumik, Code Name GOD. (Penguin Books India Pvt. Ltd., 11 Community Centre, Panchsheel Park, New Delhi 110017 , India) pp. 132-133; 161-162; 167; 171; 177; 183; 185- 186; 89-190; 198 (2006)

[5] D. Pal, Existence of thought force and its characteristics. Communicated to American Journal of Modern Physics (AJMP) (2013)

[6] V. F. Weisskopf, The Origin of the Universe, The World of Physics, (Simon and Schuster, 1230 Avenue of Americas, New York 10020) 3, pp. 10 (1987)

[7] S. W. Hawking, A Brief History of Time from the Big Bang to Black Holes. (Bantam Books, 666 fifth Avenue, New York 10103) pp. 15-36; 141-152 and 153-160 (1989)

[8] I. Prigogine and G. Nicolis, Self-Organization in NonEquilibrium Systems. (Wiley 1977) ISBN 0471024015.

[9] I. Prigogine, From Being To Becoming. (Freeman 1980). ISBN 0716711079.

[10] D. Pal, Sense of time and interpretation of the evolution of time. Communicated to American Journal of Modern Physics (AJMP) (2013)

[11] JF. Haught John, Science and Religion: From Conflict to Conversation, (Paulist Press, Mahwah, N.J., 1995) p-42 and 193

[12] L. Gilkey, Nature, Reality, and the Sacred: The Nexus of Science and Religion, (Fortress Press, Minneapolis, 1993) p89.

[13] JL. Casti, Paradigms Lost: Tackling the Unanswered
Mysteries of Modern Science, Avon, (New York, 1989) p418

[14] JA. Wheeler, "Time today", Proceedings of Workshop, "Physical origin of Time Asymmetry", (Cambridge University Press. Cambridge, U.K CB2 2RU; 1993) pp-10 to 35

[15] R. Baierlain, H. Sharp, and J. Wheeler, Three-dimensional geometry as carrier of information about time. Phys. Rev. (1962) 126,1864 to 1865

[16] C. Rovelli, "Ashtekar's formulation of general relativity and loopspace non-perturbative quantum gravity: a report", in class and QuN.Grav., (1991) Vol.8; pp-1613 to 1675.

[17] N. Birbaumer, N. Ghanayim, T. Hinterberger, I. Iversen, B. Kotchoubey, A. Kübler, J. Perelmouter, E. Taub, and H. Flor, A spelling device for the paralysed. Nature 398, Number 6725; 297-9825 (March 1999)

[18] J. P. Donoghue, L. R. Hochberg, M. D. Serruya, G. M. Friehs, J. A. Mukand, M. Saleh, A. H. Caplan, A. Branner, D. Chen, and R. D. Penn, Neuronal ensemble control of Prosthetic Devices by a Human with tetraplegia. Nature 422, 13; 164-171 (July 2000)

[19] J. P. Donoghue, Connecting cortex to machines: recent advances in brain Interfaces, Nature Neuroscience Supplement. 5 1085-8 (November 2002)

[20] J. P. Donoghue, D. Mijail, G. N. Serruya, L. P. Hatsopoulos, F. R. Matthew, Brain-machine interface: Instant neural control of a movement signal. Nature 416, 14 Number 6877 , 141 (March 2002)

[21] J. P. Donoghue, R. K. Aaron, H. M. Herr, D. McK Ciombor, L. R. Hochberg, C. L. Briant, J. R. Morgan, and M. G. Ehrlich, Horizons in prosthesis development for restoration of Limb Function. Am Accad Ortho Surg September. 14 (10) S198-S204 (2006)

[22] M. G. Hatsopoulos, D. Rubino, and K. A. Robbins, Propagating waves Mediate information transfer in the motor cortex. Nature Neuroscience. 9 1549-1557 (2006)

[23] Nicolelis MAL Miguel, Brain-Machine interfaces to restore motor function and probe neural circuits. Nature Reviews Neuroscience. 4 417-422 (2003)

[24] P. Lynds, Time and Classical and Quantum Mechanics: Indeterminacy Versus Discontinuity. Foundations of Physics Letters. 16, Number- 4, 343-355 (2003)

[25] D. D. Georgiev, Consciousness operates beyond the timescale for discerning time intervals: implications for Qmind theories and analysis of quantum decoherence in brain. NeuroQuantology 2, pp. 136 and 140-141 (2004)

[26] Vivekananda, Mâyâ and Illusion, "The complete works of Swami Vivekananda", (Advaita Ashrama, 5 Dehi Entally Road, Calcutta-700 014, 1989) 2: P-90. Mâyâ and Freedom, ibid.; 2: p-121

[27] G. Gamow, The Great Expansion. (The World of Physics. Simon and Schuster 1987) 3: p-264- 265

[28] E. Komatsu, et al. "Five-Year Wilkinson Microwave Anisotropy Probe Observations: Cosmological Interpretation". Astrophysical Journal Supplement (2009); 180 (2): 330 
[29] E. Menegoni, et al. "New constraints on variations of the fine structure constant from CMB anisotropies". Physical Review D(2009) 80 (8): 087302

[30] [30] "Origins: CERN: Ideas: The Big Bang". The Exploratorium. 2000 http://www.exploratorium.edu/origins/cern/ideas/bang.html. Retrieved 2010-09-03

[31] J. Keohane, "Big Bang theory". Ask an astrophysicist. GSFC/NASA.

http://imagine.gsfc.nasa.gov/docs/ask_astro/answers/971108 a.html.(1997). Retrieved 2010-09- 03

[32] B. J. Carr, and A. A. Coley, Persistence of black holes through a cosmological bounce. Astro- ph.co 2011;
arXiv:1104.3796v1

http://arxiv.org/abs/1104.3796

[astro-ph.CO]

[33] V. G. Gurzadyan and R. Penrose, Concentric circles in WMAP data may provide evidence of violent pre-Big-Bang activity. astro-ph.CO 2010. arXiv:1011.3706 [astro-ph.CO]

[34] Swami Vivekananda, The Vedanta Philosophy, The complete works of Swami Vivekananda, Advaita Ashrama, India, (1989) 1: pp. 360; ibid., The Cosmos, 2: pp. 206- 211; Cosmology, pp. 434-436

[35] JA. Wheeler, Beyond the End of Time. (The World of Physics. Simon and Schuster 1987) 3: p-685 